\title{
IL-17/TNF- $\alpha$ BISPECIFIC ANTIBODIES AS NEW THERAPEUTIC APPROACH TO RHEUMATOID ARTHRITIS
}

Muhammad Luthfi Adnan

Islamic University of Indonesia School of Medicine, Yogyakarta, INDONESIA

\begin{abstract}
Rheumatoid arthritis is a systemic autoimmune disease characterized by chronic inflammation causing swelling in the joints. IL17/TNF- $\alpha$ bispecific antibodies are antibodies that can bind to two different types of epitopes and work on two different types of receptors. IL-17/TNF- $\alpha$ bispecific antibodies have anti-inflammatory effects that act by blocking the inflammatory pathways of rheumatoid arthritis. Thus, bispecific antibodies have the potential to be the latest effective therapy against rheumatoid arthritis. Keywords: Bispecific antibodies, rheumatoid arthritis, therapeutics
\end{abstract}

\section{INTRODUCTION}

Rheumatoid arthritis (RA) is a systemic autoimmune disease characterized by chronic inflammation causing swelling in the joints (1). The characteristics of RA include swollen joints (commonly small joints of the hands and feet) and pain due to this swelling. On physical examination, RA presents with swellings, war$\mathrm{mth}$, and stiff joints. Initially, the pain in the joints will disappear within a few minutes, but long-term RA can cause prolonged joint pain and joint damage. These symptoms can be caused due to autoimmune reactions in synovial tissue. Damage to the joints results in physical limitations and joint pain because of the degenerative changes in cartilage (2).

\section{PATHOGENESIS OF RHEUMATOID ARTHRITIS}

Joint damage in RA starts by macrophage proliferation triggered by autoimmune factors, Porphyromonas, gingivitis infections, Epstein-Barr virus, cytomegalovirus or due to the smoking pollution of the environment. These factors can trigger the process of citrullination which is the conversion of arginine into citrulline. The presence of citrulline results in loss of tolerance in $\mathrm{T}$ cells and B cells and attacks the synovial tissue of the joints using macrophages $(3,4)$. Naive and memory B cells infiltrate the synovial tissue of the joints, resulting in continuous and further activation of B cells. Continuous activation of $\mathrm{B}$ cells produces pro-inflammatory cytokines such as tumor necrosis factor (TNF) $-\alpha$ and chemokines. Memory B cells, differentiated from immature B cells, can produce autoantibodies $(3,5)$.

$T$ cells play a role in the development of RA through T-helper (Th) 17 cells that produce IL-17 (6). Increased level of IL-17 production enhances the activity of fibroblast-like synoviocytes (FLS) which stimulate the production of matrix metalloproteinases (MMP), proinflammatory cytokines, intracellular adhesion molecule-1 (ICAM1) and vascular cell adhesion molecule-1 (VCAM-1), resulting in chronic inflammation of the joints. FLSs also activate osteoclastogenesis, a process that triggers joint damage (4). These inflammatory activities eventually cause damage to the cartilage and bone. Cartilage damage is the result of the activity of FLS to form MMP, which causes damage to connective tissue type II collagen and decreases the effectivity of inter-joint lubrication (7). Damage to the bone is the result of macrophages activating the Nucleus Factor- $\kappa$ light chain enhancer B cell (NF-KB) receptor to stimulate osteoclast activity causing bone erosion (3).

Bearing in mind its pathogenesis, RA requires articulate treatment plans that focus on its complex inflammatory reactions. Proinflammatory cytokines IL17 and TNF $\alpha$ have important roles in the development 
of rheumatoid arthritis and can be used as therapeutic targets for RA treatments.

\section{BISPECIFIC ANTIBODIES}

Bispecific antibodies (bsAbs) are antibodies that can bind to two different types of epitopes so that they can be used for different signaling (8). Bispecific antibodies can be produced by three different processes, which are; chemical conjugation, fusion of two different hybridoma lines and through recombinant DNA utilization with a genetic approach. The fusion of two different hybrids produces hybrid-hybridomas that produce heterogeneous antibodies which include bsAbs (9). However, the bsAbs can also be produced by chemical conjugation using two IgG molecules or two antigen-binding molecules (10).

The structure of bsAbs consists of 1-4 polypeptide chains which are composed of antibody components that have a component of the drug that has high-temperature reserves, high solubility, high chemicals, and low viscosity (11). Bispecific antibodies were developed from Chinese hamster ovary cells $(\mathrm{CHO})$ and Escherichia coli bacteria as host systems that have been used in biopharmaceutical technology for a long time $(11,12)$. The bsAb activity has the effect of cytotoxicity resolution through the complement system and phagocytosis to target cells through antibodies. Taking into account the mechanisms of inflammatory diseases, bsAbs have a high effect on cytokines and chemokines in vivo through targeting the inflammatory response so that it can reduce the secretion of bone reducing substances through FLS (13).

Bispecific antibodies have a short half-life due to not having a crystallized fragment that binds to the salvage receptor that allows IgG to remain at high levels (11, 14). Therefore, bsAbs are combined with a protein from the crystallized fragment $(\mathrm{Fc})$ constant in $\mathrm{IgG}$ which has an important role in the development of diseases related to interactions between proteins, ligands, and receptors and the complexity of pathological diseases. The use of a constant Fc domain of IgG which plays a role in the process of recycling endosome degradation can bind to neonatal Fc receptors (FcRn) so that bsAbs have a longer half-life and lower renal clearance (15).

The utilization of bsAbs has many advantages. Some of these advantages are; ability to bind to the appropriate target cell, having adaptable components specific to disease conditions, and capabilities obtained through an easy prokaryotic disclosure system and eukaryotes (10). High specificity is one of the most crucial advantages of bsAbs. Since the molecule can bind to two distinct receptors, it can increase the antiproliferative effect of proinflammatory cells and increase the production of proinflammatory cells simultaneously (16).

\section{BISPECIFIC ANTIBODY ACTIVITY IN RHEUMATOID ARTHRITIS}

The activation of proinflammatory cytokines IL-17 and TNF- $\alpha$ are independent of the pathogenesis of RA, however, either cytokine can be found in the joint tissue of RA patients. Both cytokines act as inflammatory mediators. Bispecific antibodies block the IL-17 and TNF- $\alpha$ pathways and reduce rheumatic symptoms in patients who have resistance to monoclonal antibody treatment. The activity of bsAbs also inhibits the production of other cytokines that are influenced by the activity of cytokine IL-17, such as IL-6 and IL-8 (17). Cytokine IL-17 pathway also plays a role in FLS activation which stimulates the production of MMP-1 and MMP-13 showing that IL-17 has a role in chronic inflammation in the joints (18).

Rheumatoid arthritis is exacerbated by the interactions of FLS and macrophages which trigger chemoattractant secretions such as Chemokine $\mathrm{C}-\mathrm{X}-\mathrm{C}$ motif Ligand 1 (CXCL1), CXCL2, CXCL6 and Chemokine C-C motif ligand (CCL) 2, resulting in synovial hypoxia. The hypoxia condition in the synovial tissue will produce hypoxia-inducible transcription factor (HIF) - 1a and will trigger the production of vascular endothelial growth factor (VEGF) which is a stimulus for angiogenesis (19).

The duality of the structure of bsAbs allows for further reduction in the inflammatory response, preventing the secondary joint damage. In addition, bsAbs play a role in preventing the production of FLS by inhibiting the activity of cytokines IL-17 and TNF- $\alpha$. Through this inhibition of FLS, chemokine production is blocked to reduce the joint damage. This method of treatment can prevent the local destruction of bone and show no adverse effect on the rest of the immune system (19). However, the findings of Xu et al. (17) show that the effects of bsAbs are not so different from the effects of monoclonal antibodies (mABs) that work with two targets. Nevertheless, using bsAbs can reduce the economic burden of RA patients since they are more affordable compared to mABs (17).

Based on the research conducted by Liu et al. (20), the use of bsAbs that target both TNF- $\alpha$ and IL-17, reduces joint destruction and inflammation induced by RA in rats compared to the use of bsAbs etanercept with dose of $3 \mathrm{mg} / \mathrm{kg}$ and etanercept dose of $3 \mathrm{mg} / \mathrm{kg}$ 
for 3 weeks. Inhibition of TNF- $\alpha$ and IL-17 pathways, also inhibit FLS formation by lowering MMP-3 levels and IL-6 levels which result in reduced osteoclast levels and their activity. Overall, this activity of molecules reduces bone erosion and maintain bone homeostasis by increased osteoblast activity and increased osteocalcin levels. Based on the research conducted by Fischer et al. (21), giving bsAbs for the treatment of RA is more effective than using a single dose of $\mathrm{mABs}$.

Bispecific antibodies are known to have better longterm therapeutic effects on RA compared to monoclonal antibodies. Findings of Alzabin et al. (22) show an increase in the severity of RA's prognosis when given a single TNF- $\alpha$ inhibitor after 15 days of discontinuation of therapy and a single IL-17 after 30 days of treatment interruption. Rats that have taken TNF- $\alpha$ and IL-17 bsAbs inhibitors can recover from RA after routine administration for 40 days and show a decrease in antigen-specific response thus providing a protective effect on the joints.

\section{CONCLUSION}

Rheumatoid arthritis is an autoimmune disease characterized with chronic inflammation of joints presenting with pain and swelling of the affected joints. Currently, therapies target the pathogenesis of RA to reduce the activity of inflammation in the joints. Bispecific antibodies can target two proinflammatory cytokines that can inhibit the activity of joint damage by proinflammatory cytokines. IL-17/TNF- $\alpha$ bispecific antibodies have higher effectiveness compared to monoclonal antibodies to achieve the cure target of rheumatoid arthritis therapy.

\section{Ethics Committee Approval: N/A \\ Informed Consent: N/A}

Conflict of Interest: The author declared no conflict of interest.

Author contributions: Concept: MLA. Supervision: MLA. Resources: MLA. Materials: MLA. Data collection and/or processing: MLA. Analysis and/or Interpretation: MLA. Literature Search: MLA. Writing Manuscript: MLA. Critical Review: MLA.

Financial disclosure: The author declared that this study received no financial support.

\section{REFERENCES}

1. Sridhar R, Ramya SS, Sowjanya S. Rheumatoid arthritis - a review. World J Pharm Pharm Sci 2016;5(10):1283-302.

2. Xu B, Lin J. Characteristics and risk factors of rheumatoid arthritis in the United States: An NHANES analysis. PeerJ 2017;24(5).

3. Mcinnes IB, Schett G. The pathogenesis of rheumatoid arthritis. N Engl J Med 2011;365(23):2205-19.

4. Cooles FA, Isaacs JD. Pathophysiology of rheumatoid arthritis. Curr Opin Rheumatol 2011;23(3):233-40.

5. Miura Y, Ota S, Peterlin M et al. Subpopulation of synovial fibroblasts leads to osteochondrogenesis in a mouse model of chronic inflammatory rheumatoid arthritis. JBMR Plus 2018;3(6):1-10.

6. Mellado M, Martinz-Munoz L, Cascio G et al. T cell migration in rheumatoid arthritis. Front Immunol 2015;6:384.

7. Bustamante MF, Garcia-Carbonell R, Whisenant KD et al. Fibroblast-like synoviocyte metabolism in the pathogenesis of rheumatoid arthritis. Arthritis Res Ther 2017;19(110):1-12.

8. Xu L, Zhang Y, Wang Q et al. Bi-specific antibodies with high antigen-binding affinity identified by flow cytometry. Int Immunopharmacol 2015;24(2):463-73.

9. Byrne H, Conroy PJ, Whisstock JC et al. A tale of two specificities: bispecific antibodies for therapeutic and diagnostic applications. Trends Biotechnol 2013;31(11):621-32.

10. Taylor P, Kontermann RE. Dual targeting strategies with bispecific antibodies. mAbs 2012;4(2):182-97.

11. Spiess C, Zhai Q, Carter PJ. Alternative molecular formats and therapeutic applications for bispecific antibodies. Mol Immunol 2015;67(2):95-106.

12. Chon JH, Zarbis-Papastoitsis G. Advances in the production and downstream processing of antibodies. N Biotechnol 2011;28(5):45863.

13. Klein C, Schaefer W, Regula JT et al. Engineering therapeutic bispecific antibodies using CrossMab technology. Methods 2019;154:21-31.

14. Michal P, Timo R, Wagne IL et al. FcRn: the architect behind the immune and non-immune functions of IgG and albumin. J Immunol 2015;194(10):4595-603.

15. Levin D, Golding B, Strome SE et al. Fc fusion as a platform technology: potential for modulating immunogenicity. Trends Biotechnol 2015;33(1):27-34.

16. Sedykh SE, Prinz VV, Buneva VN et al. Bispecific antibodies: design, therapy, perspectives. Drug Des Devel Ther 2018;12:195-208.

17. Xu T, Ying T, Wang L et al. A native-like bispecific antibody suppresses the inflammatory cytokine response by simultaneously neutralizing tumor necrosis factor-alpha and interleukin-17A. Oncotarget 2017;8(47):81860-72

18. Noack M, Beringer A, Miossec P. Additive or synergistic interactions between IL-17A or IL-17F and TNF or IL-1 $\beta$ depend on the cell type. Front Immunol 2019;10:1-12.

19. Yoshitomi H. Regulation of immune responses and chronic inflammation by fibroblast-like synoviocytes. Front Immunol 2019;10:1-8. 
20. Liu Z, Song L, Wang Y et al. A novel fusion protein attenuates collagen - induced arthritis by targeting interleukin 17A and tumor necrosis factor $\alpha$. Int J Pharm 2018;547(1-2):72-82.

21. Fischer J, Hueber A, Wilson S et al. Combined inhibition of TNFa and IL-17 as therapeutic opportunity for treatment in rheumatoid arthritis: development and characterization of a novel bispecific antibody. Arthritis Rheumatol 2015;67(1):51-62.

22. Alzabin S, Abraham SM, Taher TE et al. Incomplete response of inflammatory arthritis to TNF- $\alpha$ blockade is associated with the Th17 pathway. Ann Rheum Dis 2012;71(10):1741-8. 